\title{
Monitoring Extracellular-Vesicles Dynamics at the Nanoscale by Liquid-Cell TEM
}

Max Piffoux ${ }^{1}$, Nabeel Ahmad ${ }^{2}$, Jaysen Nelayah ${ }^{2}$, Amanda Silva ${ }^{1}$, Florence Gazeau ${ }^{1}$ and Damien Alloyeau $^{2}$

1. Laboratoire Matière et Systèmes Complexes, UMR7057 CNRS / Université Paris Diderot, Paris France.

2. Laboratoire Matériaux et Phénomènes Quantiques, UMR7162 CNRS / Université Paris Diderot, Paris, France.

Exosomes and microvesicles are promising biotherapy agents that could replace conventional cell therapies. On the road toward the use of these nano-objects in clinics, the nanoscale characterization of these complex soft materials in liquid environment has to be improved. For decades, cryo transmission electron microscopy has been a method of choice to image vesicles embedded in amorphous ice. Nevertheless the effects of freezing processes on the membrane structure remains unclear and, of course, make impossible dynamic observations. Here, we describe the use of liquid-cell transmission electron microscopy (LCTEM) for the dynamic characterization of extracellular vesicles (EV) in cellular media.

LCTEM consists in imaging the dynamics of nano-objects in an encapsulated liquid solution within an electron-transparent microfabricated cell. We exploited this in situ technique together with a goldlabeling strategy to monitor EVs in their native state with the view to explore their structural characteristics and dynamical behavior in real time with nanometer resolution. We measured relevant parameters for EV-based therapy, such as their size distribution, concentration and phosphatidyl-serine content. Besides, the morphological analyses of EV in controlled liquid environment revealed that EV deformation increases with their size and the osmotic concentration of the buffer. The deformation mechanisms in response to osmotic stress tend to transform spherical EVs (Fig 1a) to elongated vesicles corresponding to either oblate or prolate ellipsoids (Fig. 1b), or elongated vesicles with a concave (stomatocytes) or biconcave (discocyte) surface (Fig. 1c). By studying EV motion, we demonstrated that they undergo an anomalous sub-diffusion in the liquid-cell that is at least 3 orders of magnitude slower than theoretical Brownian motion, due to strong interactions with the liquid-cell windows. Interestingly, gold-labels play a key role in these EV / SiN interactions which can be efficiently used to control the diffusion of EVs, or other biomaterials, in the liquid-cell. Finally, the detection of aggregation and fusion events (Fig. 1d) illustrated that LCTEM opens up a new way to study membrane dynamics and cell processes at the nanoscale. 

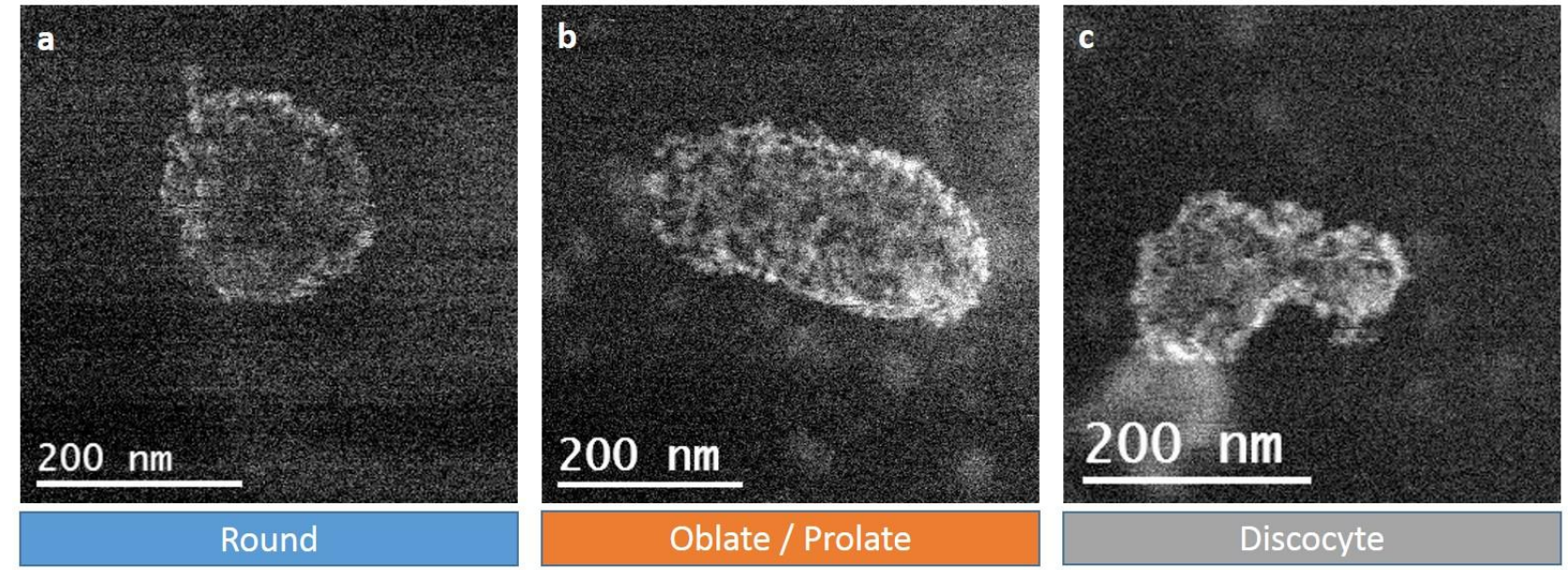

Oblate / Prolate

Discocyte

d
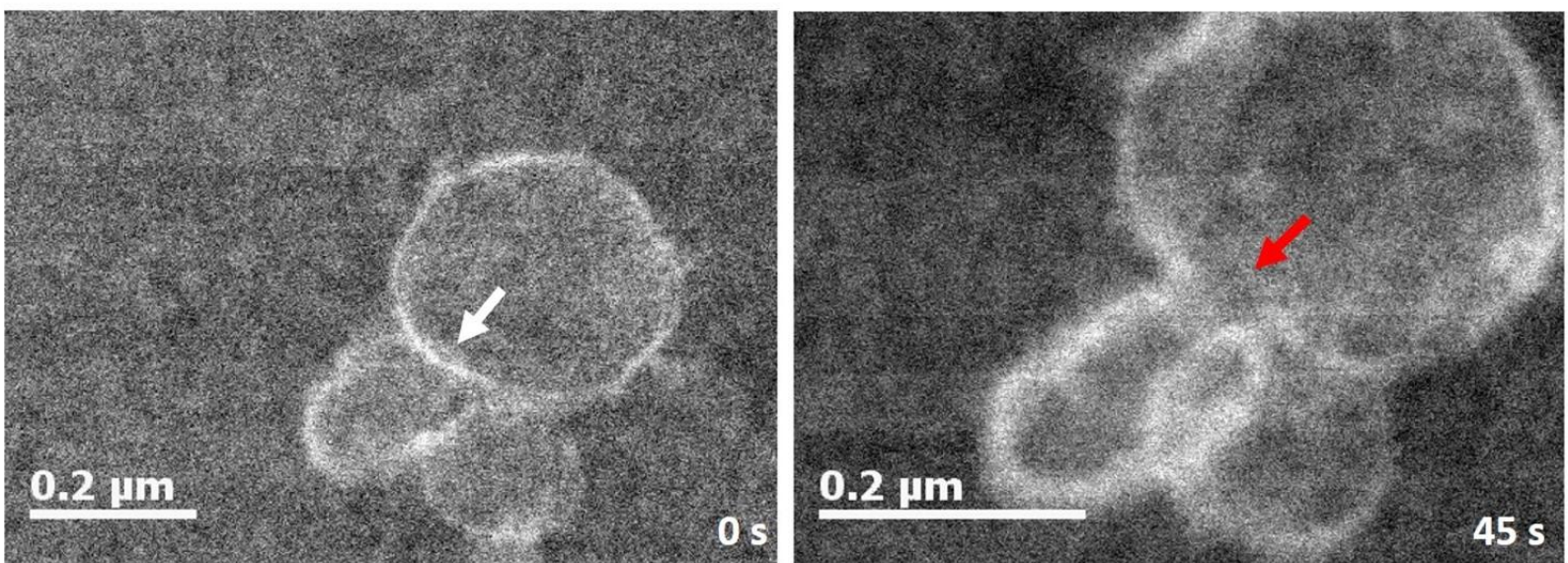

Figure 1. (a,b,c) Representative LCTEM pictures of a round EVs, an oblate (or prolate) EVs and a discocyte EVs, respectively. All the EVs are labelled with Annexin-A5 conjugated to gold nanoparticles. (d) Fusion of aggregated EVs in PBS media. The white arrow on the first image highlights the continuous interface between the two vesicles. The red arrow on the second image indicates the formation a fusion pore via the merging of the two-bilayer membranes. 